\title{
Electroanalytical biosensors for circulating tumor DNA detec- tion-A brief review
}

\author{
Zhijia Peng ${ }^{1}$, Xiaogang Lin ${ }^{1 *}$, Weiqi Nian ${ }^{2}$, Xiaodong Zheng ${ }^{2}$, Jayne Wu ${ }^{3, *}$
}

1 Key Laboratory of Optoelectronic Technology and Systems of Ministry of Education of China, Chongqing University, Chongqing, 400044, China

2 Chongqing University Cancer Hospital, Chongqing University, Chongqing, 400044, China

3 Department of Electrical Engineering and Computer Science, the University of Tennessee, Knoxville, TN 37996, USA

* Correspondence: Xiaogang Lin: xglin@cqu.edu.cn; Jayne Wu: jaynewu@utk.edu;

\begin{abstract}
Early diagnosis and treatment have always been highly desired in the fight against cancer, and detection of circulating tumor DNA (ctDNA) has recently been touted as highly promising for early cancer screening. Consequently, the detection of ctDNA in liquid biopsy gains much attention in the field of tumor diagnosis and treatment, which has also attracted research interest from the industry. However, traditional gene detection technology is difficult to achieve low cost, real-time and portable measurement of ctDNA. Electroanalytical biosensors have many unique advantages such as high sensitivity, high specificity, low cost and good portability. Therefore, this review aims to discuss the latest development of biosensors for minimal-invasive, rapid, and real-time ctDNA detection. Various ctDNA sensors are reviewed with respect to their choices of receptor probes, detection strategies and figures of merit. Aiming at the portable, real-time and non-destructive characteristics of biosensors, we analyze their development in the Internet of Things, point-of-care testing, big data and big health.
\end{abstract}

Keywords: non-destructive, biosensors, real-time detection, circulating tumor DNA (ctDNA), high sensitivity, Internet of Things.

\section{Introduction}

From the development of the first biosensor glucose sensor to the present, biosensor technology has become an interdisciplinary field of biology, chemistry, physics, medicine, electronic technology and other disciplines [1]. In recent years, biosensors are becoming an increasingly important candidate for realizing early cancer screening and tumor marker detection ${ }^{[2]}$. In the detection of tumors, traditional biopsy requires a fragment of the tumor, cannot be performed frequently, and can be rather invasive to the patient. Therefore, to make early detection of cancer possible during routine checkup, there is an urgent need for a minimal invasive, low cost and near real-time detection technology ${ }^{[3]}$.

In recent years, circulating tumor DNA (ctDNA) detection technologies have been widely studied, and was rated as one of the top ten breakthrough technologies in 2015 by MIT Magazine ${ }^{[4]}$. CtDNA is mainly composed of single or double stranded DNAs and a mixture of single and double stranded DNAs. It is around 166 nucleotides free DNA released by circulating tumor cells after necrosis and apoptosis. It exists in the peripheral blood, and can potentially act as a diagnostic biomarker with high specificity and sensitivity ${ }^{[5]}$. General ctDNA detection techniques can be divided into digital PCR ${ }^{[6]}$, droplet digital PCR [7], magnetic bead emulsion amplification [8], and label amplification depth sequencing ${ }^{[9]}$, etc. However, these technologies generally suffer from the disadvantages of high cost, poor portability, frequent occurrence of false positive and long assay time, which 
are not suitable for on-site detection and mobile medical treatment. As a contrast, ctDNA biosensor technology has high potential to be implemented for field deployable detection, because of its specificity, portability, high sensitivity and fast response [10].

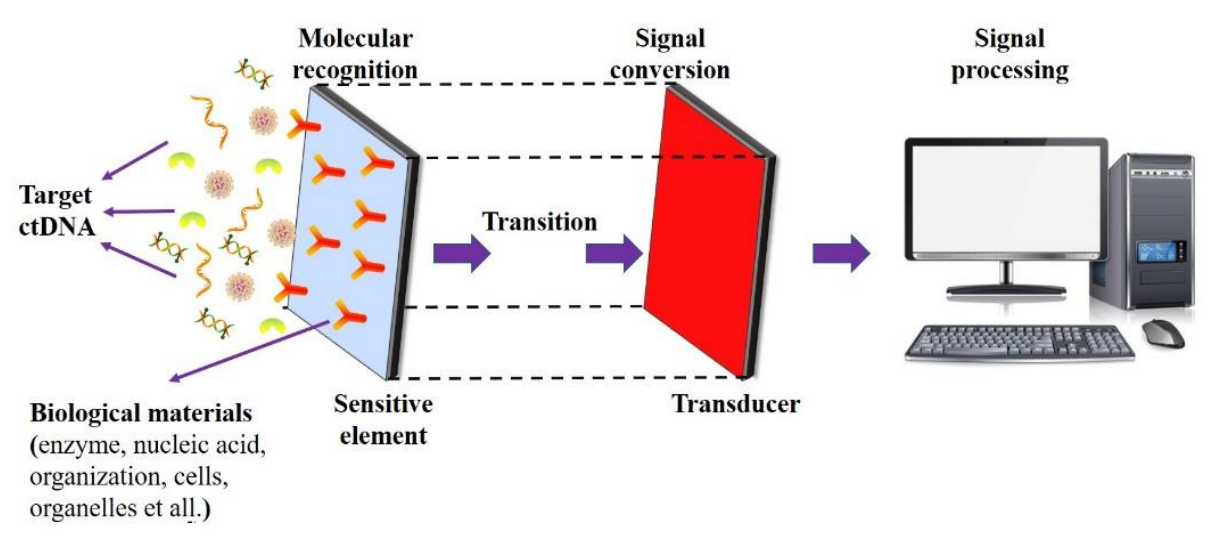

Figure 1. Schematic diagram of ctDNA biosensor detection system.

As shown in Fig.1, a ctDNA biosensor is a device that converts the biological signal generated by specific binding between the recognition probe with the target ctDNA into an electrical signal for detection, and an electroanalytical biosensor can analyze biological information from the impedance of multiple frequencies or one frequency band [11,12]. In addition, their unique advantages of low cost, portability and near real-time detection make them suitable for on-site screening ${ }^{[13]}$ and mobile health monitoring, and could become an enabling tool for personalized medicine of big data, Internet of Things and big health ${ }^{[14,15]}$.

This paper reviews the research progress of ctDNA biosensor in recent years, focusing on the types of recognition elements on the electrode surface of ctDNA biosensor. The organization is as follows: Section 2 describe the categories of recognition elements for ctDNA biosensor; Sections 3 and 4 summarizes the research progress of ctDNA electroanalytical biosensors in recent years; Section 5 discusses other types of ctDNA biosensors. Finally, the characteristics of ctDNA biosensors are summarized.

\section{Structure of surface-based biosensor}

Biosensors generally consist of two main parts: one is the biomolecular recognition element, as shown in Table 1, which has the ability of molecular recognition. Biosensors use a bioactive unit as their bio-sensitive element, which has high specificity to recognize the target analyte [16]. The biomolecular recognition elements of ctDNA biosensor can be categorized into enzymes, nucleic acids, simulated enzymes, immune substances, etc [11]. Biomolecular probes can be fixed on the surface of biosensor by physical adsorption [17], covalent bonding [18-20], embedding [21-23], self-assembled membrane ${ }^{[24-26]}$, affinity ${ }^{[27,28]}$ and other methods, as shown in Fig.2. The second part is signal conversion, namely the transducer, mainly including thermistor, electrochemical and optical detection elements [29]. They can transform the biochemical reaction into electrical signals which may be further amplified and processed to yield the concentrations of the target analytes. Therefore, a variety of surface modification techniques and different transducers can be used to construct ctDNA detection techniques. 


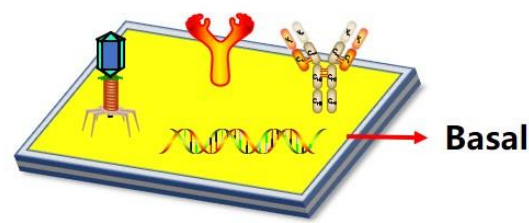

(a)Physical adsorption

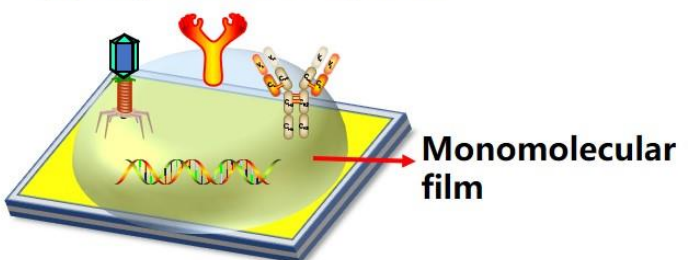

Chemical bond

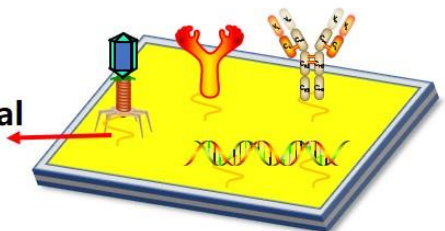

(b)Covalent bonding

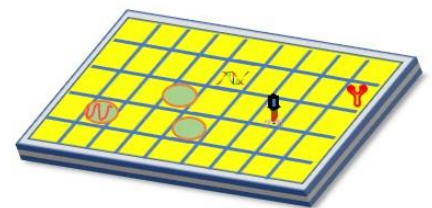

(c)Self-assembled membrane method (d)Embedding method

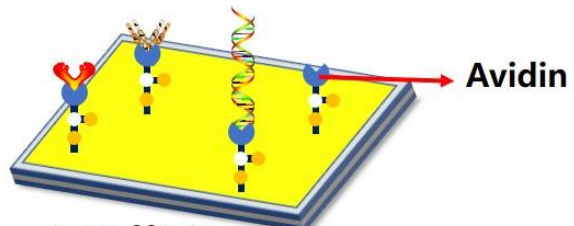

(e)Affinity

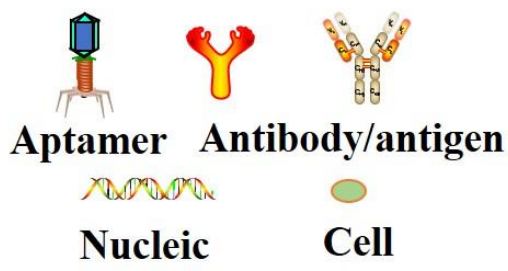

Nucleic

Cell

Figure 2. Schematic diagram of sensing surface structure.

Table 1. Biomolecular recognition devices.

\begin{tabular}{cc}
\hline Biosensitive element & Bioactive unit \\
\hline Enzyme & Varity of enzymes \\
\hline Nucleic acid & DNA, RNA, etc. \\
\hline Organization & Tissue sections of plants and animals \\
\hline Cells & The cells of bacteria, fungi, plants, and animals \\
\hline Organelles & Mitochondria, chloroplasts \\
\hline Simulation of enzyme & Macromolecule polymer \\
\hline Immune substances & Antigens, antibodies, etc. \\
A substance with biological affinity & Ligand, receptor \\
\hline
\end{tabular}

At present, clinical detection of ctDNA is mainly based on polymerase chain reaction (PCR) technology and gene sequencing, while there are few reports on ctDNA biosensors, partly due to the small sizes of ctDNA. Compared with PCR and gene sequencing, ctDNA biosensors are of lower cost, higher sensitivity, and are easier to integrate and carry, making them more suitable for on-site detection and mobile medical development.

In the following, different biosensing approaches applied to ctDNA detection are discussed based on the existing ctDNA biosensor reports.

\section{Nuclei acid probe-based detection}

Nuclei acid identification biosensors are widely used in clinical diagnosis [30,31], microbial detection ${ }^{[32]}$ and environmental monitoring [33] because of their high sensitivity, fast response, simple operation, low price and miniaturization. The probe can recognize the target DNA molecule by specific hybridization and transform the reaction into electrical signal for analysis. 

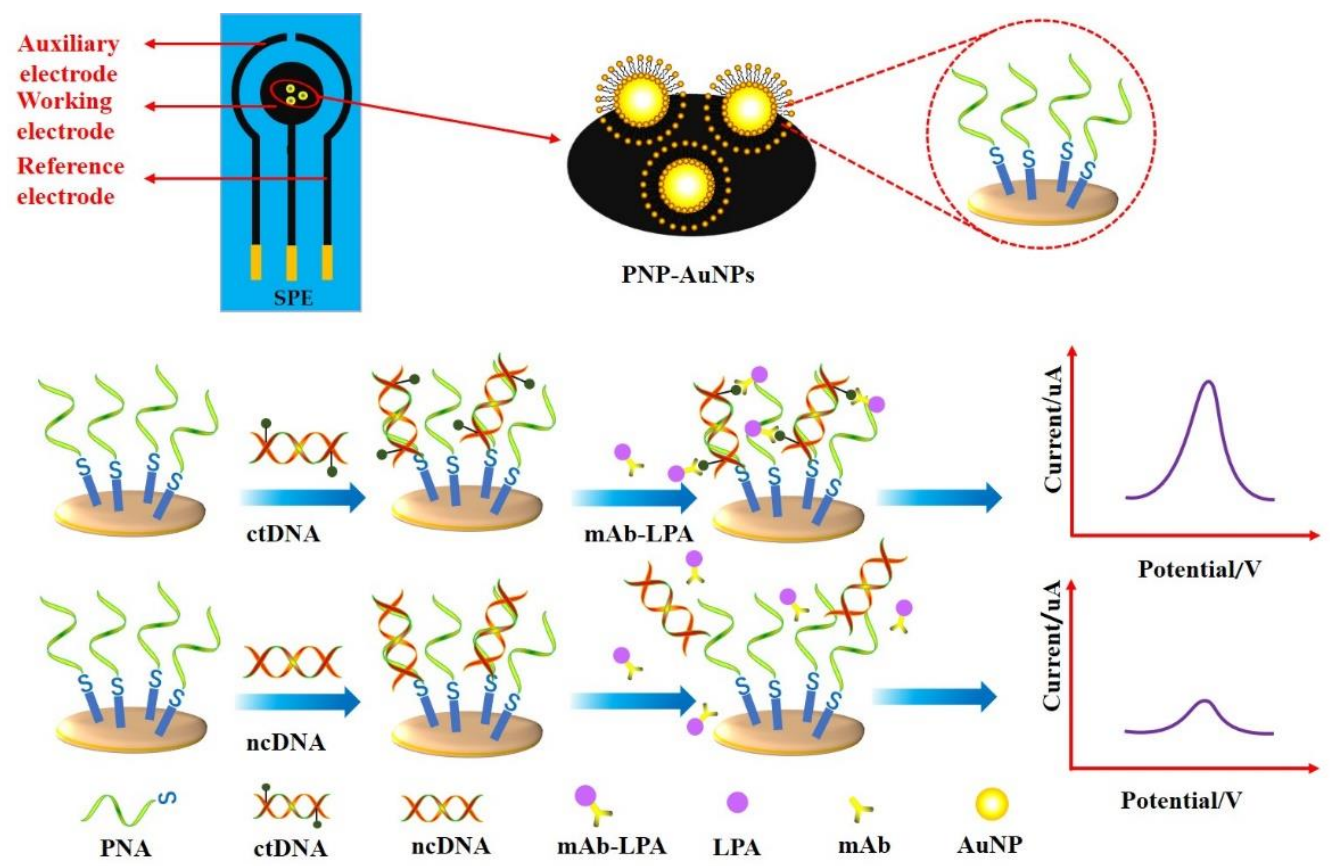

Potential/V

Figure 3. Schematic illustration of DNA electrochemical biosensor and ctDNA detection. The PNA probe was fixed with gold nanoparticles on the working electrode. PNA will bind to ctDNA with a specific form. At the same time, mAb-LPA will specifically bind to ctDNA to release potential, but not to ncDNASchematic diagram of sensing surface structure.

At present, DNA sensors mainly use peptide nucleic acid (PNA) as a probe to specifically bind ctDNA, to capture the target DNA. Since there is no electrostatic repulsion between PNA and DNA, the ability of PNA to hybridize with DNA molecules is superior to DNA/DNA. The PNA probe is fixed on the surface of the sensor using covalent bond modification technology, that is, the sulfhydryl group of PNA forms an Au-S bond with the gold electrode, so that the PNA is immobilized on the electrode surface. Chunyan et al. ${ }^{[34]}$ proposed a dual biomarker detection platform based on a PNA probe-gold nanoparticles and lead phosphate apoferritin for the detection of PIK3CA gene tumor characteristic mutation and methylation, in order to quantify ctDNA, which is described in Fig. $3{ }^{[34]}$. The DNA methylation refers to the DNA methylation transfer enzyme under the action of the genome $\mathrm{CpG}$ dinucleotide cytosine 5 carbon covalent bond combined with a methyl group.

Firstly, a large amount of PNA was fixed on the surface of a screen-printed electrode (SPE) with gold nanoparticles as the carrier through covalent bonds. PNA probe can form stable complexes with DNA though complementary base pairing or Hoogsteen base pairing principle ${ }^{[3,36]}$. When a solution of ctDNA with mutant sites was dropped onto the SPE's working electrode, the mutant sites bound to the PNA probe in a complementary and specific way. Afterwards, monoclonal antibody carrier lead phosphate apoferritin (LPA) solution was added, which could amplify the selectivity of ctDNA, thus enhancing the electrochemical detection signal. In contrast, when normal ctDNA (ncDNA) solution was dropped to the electrode, ncDNA could neither bind complementary to the PNA probe, nor bind to mAb-LPA, resulting in almost no potential change. Compared to normal cells, the genetic changes after cell carcinoma were the methylation level of the anticancer gene increased, and the methylation level of the primary cancer gene decreased. Therefore, abnormal levels of ctDNA methylation also can be used to verify whether a tumor is present in the body. This new method based on DNA probe complementation had excellent selectivity and ultra-high sensitivity, and the detection limit is $1.0 \times 10^{-14}$ $\mathrm{mol} / \mathrm{L}$. 
To further improve the selectivity of PNA probe based ctDNA sensor, Das et al. [37] designed a DNA clutch probe to prohibit the recombination of ssDNA stands. The PNA probe was fixed on the gold electrode by covalent bonding method, which specifically bound the mutated target gene ssDNA, resulting in the change of electrode potential. Potential changes were detected to reflect the mutation of ctDNA in serum.

\subsection{DNA probe}

For the detection of ctDNA, Mahbubur et al. ${ }^{[38]}$ modified graphene oxide coated gold nanostar onto the glassy carbon electrode and fixed the target probe through the $\pi-\pi$ interaction between DNA bases to detect ctDNA in the peripheral blood of gastric cancer. The hybridization of the probe DNA with ctDNA caused the resulting dsDNA to detach from the electrode's surface, leading to an increase of electrical current. The detection limit was as low as $1.0 \times 10^{-20} \mathrm{~mol} / \mathrm{L}$. This method was used to analyze ctDNA in serum samples of cancer patient, which has great potential for the application of ctDNA detection in realtime detection research.

Wei Zhange et al. ${ }^{[39]}$ established a $\mathrm{MoS}_{2}$ nanosheet polymer biosensor based on poly-xanthurenic acid film functionalization. As described in Fig.4 [39], this polymer biosensors were electropolymerized by Poly-xanthurenic acid (PXA) on the surface of the $\mathrm{MoS}_{2}$ electrode prepared in advance, thus forming an ideal interface for the PIK3CA gene expressed in the peripheral blood of patients with gastric cancer. Then the probe ssDNA was directly fixed on the PXA/ $\mathrm{MoS}_{2}$ nanocomposite, and the change of self-signal after ssDNA hybridization with target DNA was induced by cyclic voltammetry and electrochemical impedance spectroscopy ${ }^{[39]}$. The limit of detection of the polymer biosensor was reported to be $1.8 \times 10^{-17} \mathrm{~mol} / \mathrm{L}$ for the PIK3CA gene ${ }^{[39]}$.
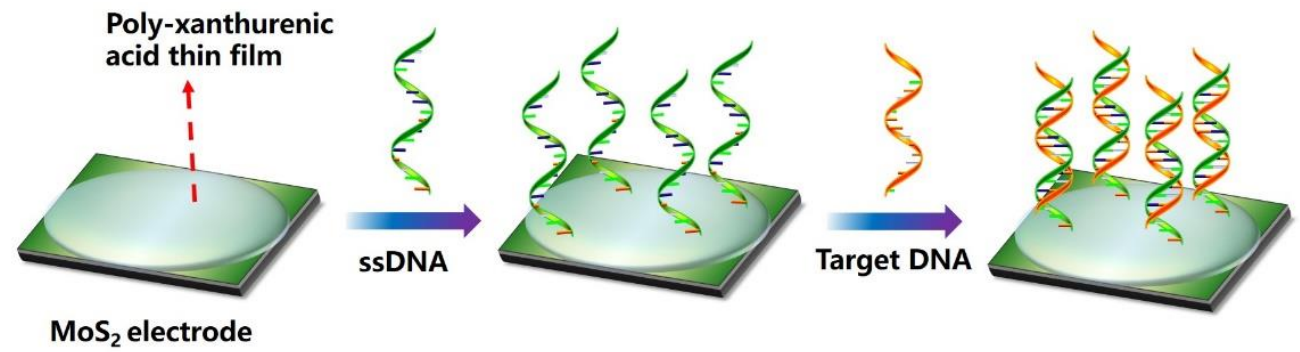

Figure 4. Schematic display of high-performance polymer biosensor based on poly-xanthurenic acid functionalized $\mathbf{M o S}_{2}$ nanosheets.

In 2018, Wang HF et al. [40] designed a multifunctional label-free circulating tumor DNA KRAS G12DM enzyme electrode biosensor based on the terminal deoxynucleotide transferase (TdT) and ribonucleic acid HII (RNase HII) dual-enzyme co-group amplification strategy. As shown in the Fig. $5^{[40]}$, the biosensor consisted of a triple-helix molecular switch (THMS) as molecular recognition and signal transduction probe, ribonuclease HII (RNase HII) and terminal deoxynucleotidyl transferase (TdT) as dual enzyme assisted multiple amplification accelerator ${ }^{[41]}$. Signal transduction probe (STP) is released under the action of RNase HII, and the capture probe fixed on the gold electrode is hybridized with STP, and then TdT is used to achieve TdT-mediated cascade expansion to generate a stable DNA dendritic structure. Finally, the electrically active molecule methylene blue $(\mathrm{MB})$ was obtained. The labeled electroactive tree achieved highly sensitive and accurate detection of ctDNA. The limit of detection was down to aM [40]. In the same way, Zhou Q et al. ${ }^{[1]}$ proposed a new design idea, construction of SWNT-based SERS assay coupling with RNase HII-assisted amplification for highly sensitive detection of ctDNA in human blood. The limit of detection of the new method was reported to be 3.0 $\times 10^{-16} \mathrm{~mol} / \mathrm{L}$ for the KRAS G12DM gene. 


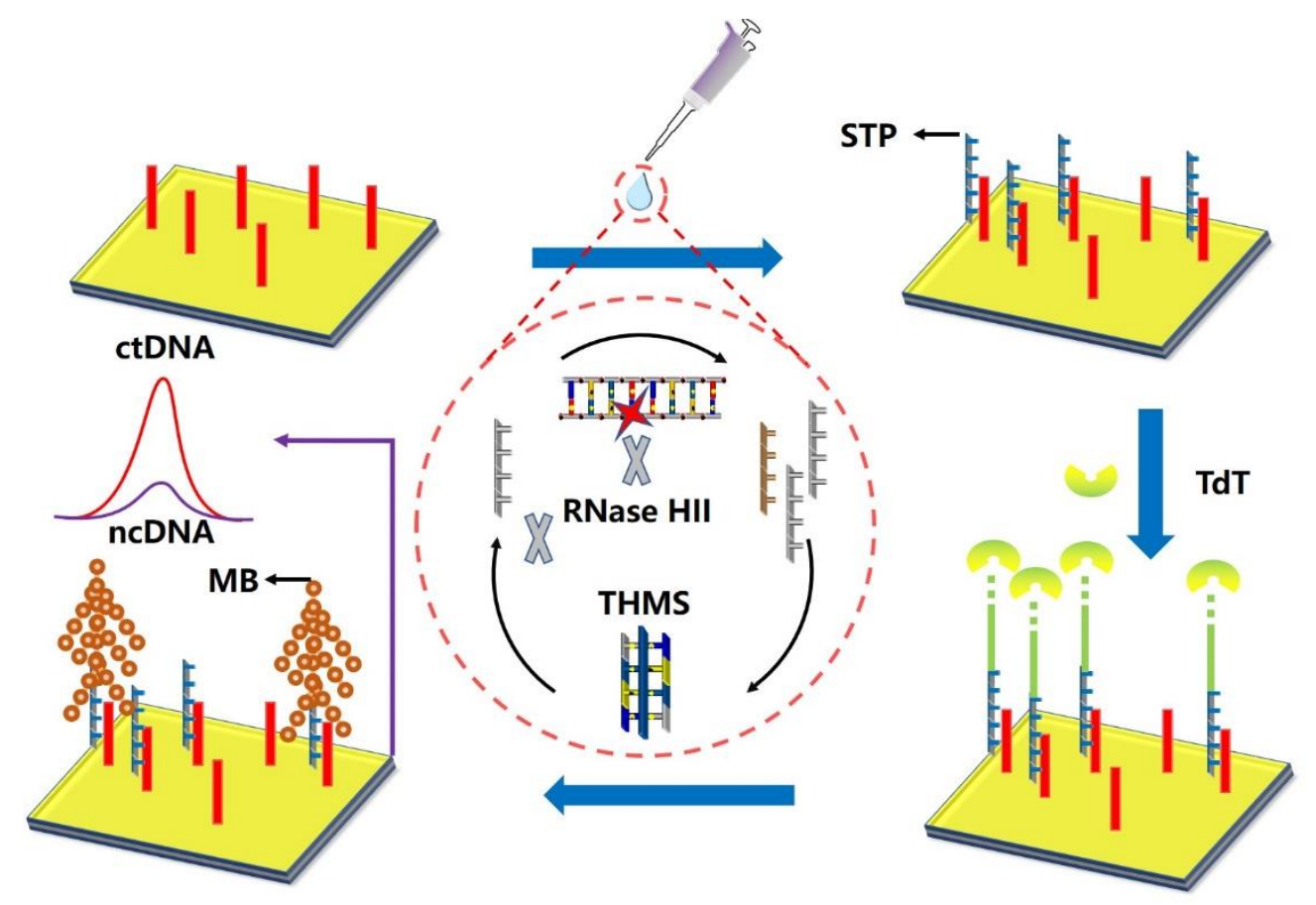

Figure 5. Schematic illustration of the enzyme biosensor using THMS probe and TdT and RNase HII dual amplification.

\section{Antibody probe-based detection}

Antibody have been widely used in disease diagnosis and treatment, food safety control and environmental monitoring ${ }^{[42]}$. Specific binding by antibody is the foundation for the detection of pathogens, micro molecules, cells, bacteria, and other molecules with biological characteristics. Detection based on antibody probe has the advantages of reducing non-specific interference and lowering the lower limit of detection. Antibody specific to target ctDNA is immobilized on the electrodes. Captured ctDNA was detected by electrochemical method or enzyme-linked immunosorbent assay.

At present, DNA site-specific methylation has been increasingly used as a biomarker ${ }^{[43-45]}$. In addition, ctDNA methylation is an important epigenetic modification to control tumors, and detection of ctDNA methylation level can effectively determine the degree of malignancy of tumors ${ }^{[46]}$. The methods for analyzing ctDNA methylation mainly include PCR, sequencing and microarray, etc., but all of these methods require ctDNA pretreatment ${ }^{[77,48]}$. In contrast, 5-methylcytosinine (5-MC) monoantibodies can be directly immobilized on the electrode by covalently coupling method, which can be used to capture methylated ctDNA without sample pretreatment. In 2018, Povedano et al. [49] proposed to use a 5-mC single antibody as a ctDNA receptor, and used a hydrogen peroxide/hydroquinone $(\mathrm{H} 2 \mathrm{O} 2 / \mathrm{HQ})$ system to process screen-printed carbon electrodes (SPCE) for ampere testing. As shown in Fig. ${ }^{[49]}$, this immunosensor used two different antibodies. The first of them is a 5 -mC antibody immobilized on the surface of carboxylic acid-modified magnetic beads, capable of capturing any ssDNA with ctDNA methylation sequence. The second was an antibody labeled with peroxidase (HRP-anti-ssDNA) as a signaling antibody, recognizing any ssDNA [49]. In 2019, Povedano et al. ${ }^{[50]}$ reported that their ctDNA biosensor was able to detect methylation in RNA with a lower limit of $1.25 \times 10^{-15} \mathrm{~mol} / \mathrm{L}$ without changing the modified antibody of the sensor. Compared with traditional methods, the sensor electrode physically adsorbs the antigen, which is simple to operate and low in cost, and can specifically identify ctDNA methylation. Therefore, 
the sensor strategy is more suitable for applications in the field of real-time detection and mobile health care.

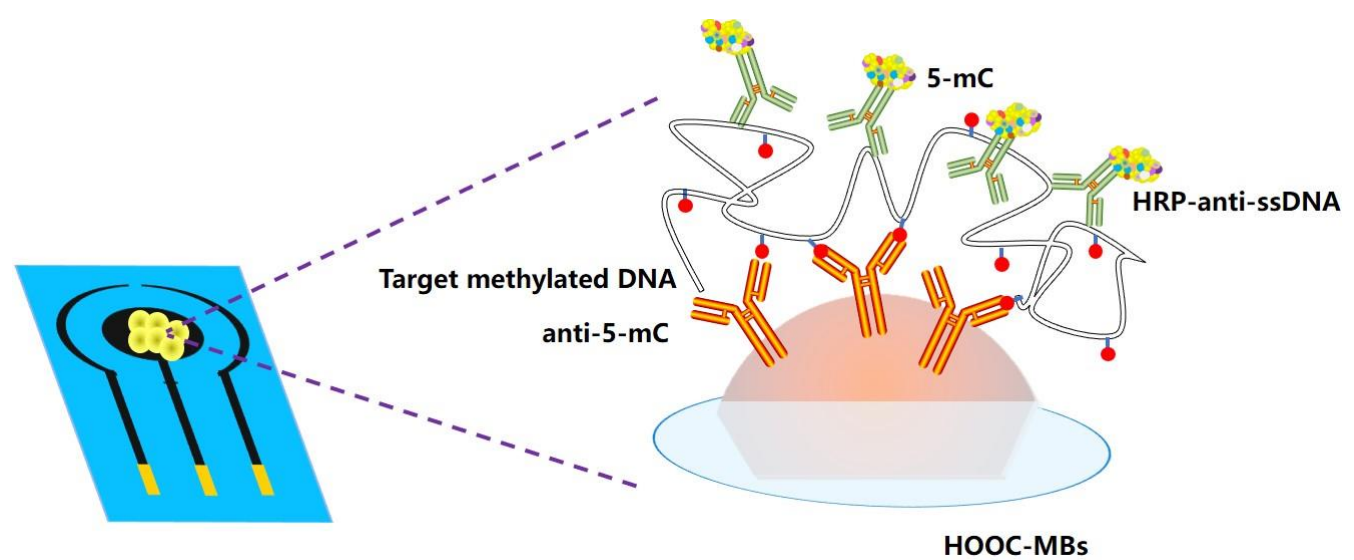

Figure 6. Schematic display of the immunosensor and ctDNA detection.

\section{Other ctDNA biosensing methods}

For the completeness of the discussion on ctDNA biosensors, the optical sensing methods of ctDNA are briefly presented as follows. Chang et all. ${ }^{[51]}$ proposed a fluorescence polarization method to detect ctDNA based on DNA dissociation probe and chain exchange mechanism, and used toehold-mediated strand displacement reaction (TSDR) to identify the specific binding of probes to target mutated DNA to generate fluorescence for qualitative analysis. Jin J et all. ${ }^{[52]}$ designed and synthesized a water-soluble cationic fluorescent probe, and found that fluorescence intensity was linearly proportional to ctDNA concentration at a certain concentration. Especially important, Li R et all. [53] proposed an amplified colorimetric biosensor for ctDNA. The colorimetric biosensor utilized the amplification property of HCR and the high peroxidase with asymmetric catalytic activity to split G-quadruplex DNA zymes, with produces color signals in the presence of ctDNA, and has been successfully applied in complex biological environments such as human blood plasma for ctDNA detection, with the detection limit corresponding to 1.0 $\times 10^{-10} \mathrm{~mol} / \mathrm{L}^{[59]}$. The realization of ctDNA detection based on fluorescent and colorimetric provides valuable theoretical knowledge for ctDNA biosensors in the field of real-time detection and mobile health.

Although the detection method based on ctDNA optical reaction can be better used in mobile medicine, its detection limit can only reach pM level. CtDNA detection methods based on electrochemistry have higher sensitivity and detection limits can almost reach the level of $\mathrm{fM}$, as shown in Table 2.

Table 2. Comparison of different methods.

\begin{tabular}{ccc}
\hline Methods & Level & Reference \\
\hline $\begin{array}{c}\text { Electrochemical method based on } \\
\text { DNA probe }\end{array}$ & $\mathrm{fM}$ & {$[34],[38],[39],[41],[42]$} \\
\hline $\begin{array}{c}\text { Electrochemical method based on an- } \\
\text { tigen antibody }\end{array}$ & $\mathrm{fM}$ & {$[51],[52]$} \\
\hline Fluorescence method & $\mathrm{pM}$ & {$[57],[58]$} \\
\hline Amplified colorimetric method & $\mathrm{pM}$ & {$[59]$} \\
\hline
\end{tabular}

\section{Conclusions and Prospects}

This paper reviews the new advances in electroanalytical biosensors for the detection of ctDNA. Among the traditional and common clinical technologies for ctDNA detection, 
PCR and DNA sequencing are mainly used, but these technologies require expensive instruments, complex operations, low sensitivity and high false positive, which are not suitable for point-of-care testing and Internet of Things. Sensitive and specific biosensors with simpler operation and real-time monitoring will become a powerful tool for the detection of ctDNA. As biosensors can be easily made into portable and low-cost devices, the biosensors will become a trend of the new technological revolution in ctDNA detection and point-of-care testing. This review mainly analyzes the detection of ctDNA by electroanalytical biosensors with details on the strategies of sensor modification and signal amplification. In the end, this paper introduces the other biosensing methods for ctDNA detection. At present, the implementation of this method in the field of precision medicine and mobile health is full of opportunities and challenges, with many challenges to be overcome.

Author Contributions: Methodology, formal analysis, investigation, data curation, writing - original draft, Z.P.; conceptualization, validation, writing - review and editing, supervision, project administration, funding acquisition, X.L, X.Z.; resources, writing-original draft, Q.N., J.W.

Funding and Acknowledgments: This research was funded by the Fundamental Research Funds for the Central Universities (Project No.2019CDYGZD006), National Natural Science Foundation of China (Project no. 6137 7001), the Postgraduate education and teaching reform research project of Chongqing University (Project No. cquyjg18323), Venture \& Innovation Support Program for Chongqing Overseas Returnees and Fundamental Research Funds for the Central Universities (No. 10611CDJXZ238826).

Conflicts of Interest: The authors declare no conflict of interest. The funders had no role in the design of the study; in the collection, analyses, or interpretation of data; in the writing of the manuscript, or in the decision to publish the results.

\section{References}

1. Singh R P. Prospects of Nano biomaterials for Biosensing[J]. International Journal of Electrochemistry, 2011, 2011(4).

2. Pollard TD, Ong JJ, Goyanes A, et al. Electrochemical biosensors: a nexus for precision medicine - ScienceDirect[J]. Drug Discovery Today, 2020,26(1):69-79.

3. Lehmann, Bartels. [Liquid biopsy in tumor diagnostics: Applications, perspectives, and limitations of the "cancer liquidome"]. [J]. Der Pathologe, 2019.

4. Zhang SQ, Huang WQ. Circulating tumor DNA and liquid biopsy in early screening of gynecologic tumor. Chinese Journal of Practical Gynecology and Obstetrics, 2016, 32(5): 405-409.

5. Zou Z, Qi P, Qing Z, et al. Technologies for analysis of circulating tumour DNA: Progress and promise[J]. TrAC Trends in Analytical Chemistry, 2017:S0165993617301875.

6. Naoki, Takegawa, Kimio, et al. HER2 genomic amplification in circulating tumor DNA from patients with cetuximab-resistant colorectal cancer[J]. Oncotarget, 2015, 7(3):3453-3460.

7. Tsao $\mathrm{CH}$, Weiss J, Hudson $\mathrm{C}$, et al. Monitoring response to therapy in melanoma by quantifying circulating tumor DNA with droplet digital PCR for BRAF and NRAS mutations[J]. Scientific Reports, 2015, 5: 11198.

8. Forthun RB, Hovland R, Schuster C, et al. ctDNA detected by ddPCR reveals changes in tumor load in metastatic malignant melanoma treated with bevacizumab[J]. Scientific Reports, 2019, 9(1):17471.

9. Gale D, Plagnol V, Lawson A, et al. Abstract 3639: analytical performance and validation of an enhanced TAm-Seq circulating tumor DNA sequencing assay [J]. Cancer Research, 2016, 76(14):3639.

10. Sharifi M, Hasan A, Attar F, et al. Point-of-care nanobiosensor in breast cancers[J]. Talanta, 2020.

11. Turner A P. Advances in Biosensors. JAI Press Ltd, 2003.

12. Antiochia R. Developments in biosensors for $\mathrm{CoV}$ detection and future trends[J]. Biosensors and Bioelectronics, 2020, 173:112777.

13. The Current Situation and Development Prospect of POCT[J]. Clinical Laboratory Journal (Electronic Edition), 2015.

14. Li X, Ye M, Zhang W, et al. Liquid biopsy of circulating tumor DNA and biosensor applications[J]. Biosensors \& Bioelectronics, 2019, 126:596-607.

15. Das J, Kelley SO. High-Performance Nucleic Acid Sensors for Liquid Biopsy Applications[J]. Angewandte Chemie International Edition, 2020, 59(7):2554-2564.

16. Chambers J P, Arulanandam B P, Matta L L, et al. Biosensor recognition elements[J]. Current issues in molecular biology, 2008, 10(1-2):1-12.

17. Voss S, Newman E, Miller-Schulze JP. Quantification of Sucralose in Groundwater Well Drinking Water by Sialylation Derivatization and Gas Chromatography-Mass Spectrometry[J]. Analytical Methods, 2019, 11(21):2790-2799. 
18. Jiang B, Yu H, Zhang Y, et al. A multiarticulate delivery system for potential colonic targeting using bovine serum albumin as a model protein[J]. Pharmaceutical Research,2017,34(12):1-12.

19. Armbruster CR, Wolter DJ, Mishra M, et al. Staphylococcus aureus protein a mediates interspecies interactions at the cell surface of pseudomonas aeruginosa[J]. mBio,2016,7(3):2-16.

20. Hiebl B, Ascher L, Luetzow K, et al. Albumin solder covalently bound to a polymer membrane: new approach to improve binding strength in laser tissue soldering in-vitro[J]. Clinical Hemorheology and Microcirculation,2018,69(3):1-10.

21. Stan CS, Horlescu G, Petronela, et al. Facile preparation of highly luminescent composites by polymer embedding of carbon dots derived from N-hydroxy phthalimide[J]. Journal of Materials Science, 2017, 52(1):185-196.

22. Kulicek J, Gemeiner P, Omastová M, et al. Preparation of polypyrrole/multi-walled carbon nanotube hybrids by electro polymerization combined with a coating method for counter electrodes in dye-sensitized solar cells[J]. Chemical Papers- Slovak Academy of Sciences, 2018, 72(7):1651-1667.

23. Hassanein A, Salahuddin N, Matsuda A, et al. Fabrication of electrochemical sensor based on layered double hydroxide/polypyrrole/carbon paste for determination of an alpha-adrenergic blocking agent terazosin[J]. Electroanalysis,2018,30(3):236-351.

24. Cui C, Deng Y, Han L. Bicontinuous cubic phases in biological and artificial self-assembled systems[J]. Science China Materials, 2020, 63(5):686-702.

25. Luo H, X Lin, Peng Z, et al. Rapid and Sensitive Detection of Bisphenol A Based on Self-Assembly[J]. Micromachines, 2020, $11(1), 41$.

26. Dey K. Self-Assembly-Driven Nano mechanics in Porous Covalent Organic Framework Thin Films[J]. Journal of the American Chemical Society, 2021, 143(2), 955-963.

27. Kim H, Tran MV, Petryayeva E, et al. Affinity Immobilization of Semiconductor Quantum Dots and Metal Nanoparticles on Cellulose Paper Substrates[J]. ACS Applied Materials \& Interfaces, 2020, 12(47):53462-53474.

28. Bormann S, Burek BO, Ulber R, et al. Immobilization of unspecific peroxygenase expressed in Pichia pastoris by metal affinity binding[J]. Molecular Catalysis, 2020, 492.

29. Yang Y, Yang X, Yang Y, et al. Aptamer-functionalized carbon nanomaterials electrochemical sensors for detecting cancer relevant biomolecules[J]. Carbon, 2018, 129:380-395.

30. Yao U, Hao P, Jan, et al. Clicking DNA to gold nanoparticles: poly-adenine-mediated formation of monovalent DNA-gold nanoparticle conjugates with nearly quantitative yield[J]. Npg Asia Materials 7, e159,2015.

31. Fan, Yang, Xiaolei, et al. A Bubble-Mediated Intelligent Microscale Electrochemical Device for Single-Step Quantitative Bioassays[J]. Advanced Materials, 2014, 26(27):4671-4676.

32. Zhilei GE, Pei H, Wang LH, et al. Electrochemical single nucleotide polymorphisms genotyping on surface immobilized threedimensional branched DNA nanostructure[J]. Science China Chemistry, 2011, 54(008):1273-1276.

33. Lapierre-Devlin MA, Asher CL, Taft BJ, et al. Amplified Electrocatalysis at DNA-Modified Nanowires[J]. Nano Letters, 2005, 5(6):1051-1055.

34. Cai C, Guo Z, Cao Y, et al. A dual biomarker detection platform for quantitating circulating tumor DNA (ctDNA)[J]. Nanotheranostics, 2018, 2(1):12-20.

35. Boffa LC, Carpaneto EM, Allfrey VG. Isolation of active genes containing CAG repeats by DNA strand invasion by a peptide nucleic acid[J]. Proceedings of the National Academy of Sciences, 1995, 92(6): 1901-1905.

36. Moccia M, Antonacci A, Saviano M, et al. Emerging technologies in the design of peptide nucleic acids (PNAs) based biosensors[J]. TrAC Trends in Analytical Chemistry, 2020, 132:116062.

37. Das J, Ivanov I, Sargent EH, et al. DNA clutch probes for circulating tumor DNA analysis[J]. Journal of the American Chemical Society, 2016, 138(34): 11009-11016.

38. Rahman M, Cui D, Zhou S, et al. A graphene oxide coated gold nanostar based sensing platform for ultrasensitive electrochemical detection of circulating tumor DNA[J]. Analytical Methods, 2020, 12(4): 440-447.

39. Zhang W, Dai Z, Liu X, et al. High-performance electrochemical sensing of circulating tumor DNA in peripheral blood based on poly-xanthurenic acid functionalized MoS2 nanosheets[J]. Biosensors \& Bioelectronics, 2018, 105:116.

40. Hua-Feng, Wang, Rong-Na, et al. A versatile label-free electrochemical biosensor for circulating tumor DNA based on dual enzyme assisted multiple amplification strategy[J]. Biosensors \& Bioelectronics, 2018; 122:224-230.

41. Zhou Q, Zheng J, Qing Z, et al. Detection of Circulating Tumor DNA in Human Blood via DNA-Mediated Surface-Enhanced Raman Spectroscopy of Single-Walled Carbon Nanotubes[J]. Analytical Chemistry, 2016; 88(9):4759-4765.

42. Kano K, Shirai O, Y Kitazumi, et al. Applications to Biosensors[J]. 2021.

43. Warton K, Mahon KL, Samimi G. Methylated circulating tumor DNA in blood: power in cancer prognosis and response[J]. Endocrine-related cancer, 2016, 23(3): R157-R171.

44. Mishima C, Kagara N, Matsui S, et al. Promoter methylation of TRIM9 as a marker for detection of circulating tumor DNA in breast cancer patients[J]. Springer plus, 2015, 4(1): 635 .

45. Balgkouranidou I, Chimonidou M, Milaki G, et al. SOX17 promoter methylation in plasma circulating tumor DNA of patients with non-small cell lung cancer[J]. Clinical Chemistry and Laboratory Medicine (CCLM), 2016, 54(8): 1385-1393.

46. Whalley C, Payne K, Domingo E, et al. Ultra-Low DNA Input into Whole Genome Methylation Assays and Detection of Oncogenic Methylation and Copy Number Variants in Circulating Tumour DNA[J]. Epigenomes, 2021, 5(1):6.

47. Khatami F, L Teimoori Oolabi, R Heshmat, et al. Circulating ctDNA methylation quantification of two DNA methyl transferases in papillary thyroid carcinoma[J]. Journal of Cellular Biochemistry, 2019, 120(1). 
48. Syedmoradi L, Esmaeili F, Norton M L: Towards DNA methylation detection using biosensors. Analyst, 2016 ; $141: 5922-43$.

49. Povedano E, Vargas E, Montiel VR et al: Electrochemical affinity biosensors for fast detection of gene-specific methylations with no need for bisulfite and amplification treatments. Sci Rep, 2018; 8: 6418.

50. Povedano E, Montiel VR, Valverde A et al: Versatile electroanalytical bio-platforms for simultaneous determination of cancerrelated DNA 5-meth-yl- and 5-hydroxymethyl-cytosines at global and gene-specific levels in human serum and tissues. ACS Sens, 2019; 4: 227-34.

51. Chang H, Zhang Y, Yang F, et al. ctDNA Detection Based on DNA Clutch Probes and Strand Exchange Mechanism[J]. Frontiers in Chemistry, 2018, 6:530.

52. J, Chen X, Liu Y, et al. Detection of ctDNA with water soluble tetraphenylene-based fluorescence probe[J]. Acta Polymeric a Sinical, 2011; 011(9):1079-1085.

53. Li R, Zou L, Luo Y, et al. Ultrasensitive colorimetric detection of circulating tumor DNA using hybridization chain reaction and the pivot of triplex DNA[J]. Scientific Reports, 2017, 7:44212. 